\title{
Near-surface stellar magneto-convection: simulations for the Sun and a metal-poor solar analog
}

\author{
Matthias Steffen ${ }^{1}$, H.-G. Ludwig ${ }^{2}$, and O. Steiner ${ }^{3}$ \\ ${ }^{1}$ Astrophysikalisches Institut Potsdam, An der Sternwarte 16, D-14482 Potsdam, Germany, \\ email: msteffen@aip.de \\ ${ }^{2}$ GEPI, Observatoire de Paris, CNRS, Université Paris Diderot, 92195 Meudon Cedex, France \\ ${ }^{3}$ Kiepenheuer-Institut für Sonnenphysik, Schöneckstrasse 6, D-79104 Freiburg, Germany
}

\begin{abstract}
We present 2D local box simulations of near-surface radiative magneto-convection with prescribed magnetic flux, carried out with the MHD version of the $\mathrm{CO}^{5} \mathrm{BOLD}$ code for the Sun and a solar-like star with a metal-poor chemical composition (metal abundances reduced by a factor $100,[\mathrm{M} / \mathrm{H}]=-2$ ). The resulting magneto-hydrodynamical models can be used to study the influence of the metallicity on the properties of magnetized stellar atmospheres. A preliminary analysis indicates that the horizontal magnetic field component tends to be significantly stronger in the optically thin layers of metal-poor stellar atmospheres.
\end{abstract}

Keywords. Convection - Sun: atmosphere - stars: atmospheres - MHD

\section{Introduction}

Up to now, numerical simulations of near-surface magneto-convection are essentially restricted to the Sun (see e.g. Carlsson et al. 2004; Vögler et al. 2005; Stein \& Nordlund 2006; Schüssler \& Vögler 2008; Steiner et al. 2008). The idea of the present work is to study the influence of metallicity on the structure and distribution of magnetic flux concentrations in stellar atmospheres. For this purpose, realistic local box simulations of near surface magneto-convection with prescribed magnetic flux have been carried out for the Sun and a metal-poor solar analog, allowing a differential comparison of the thermal, dynamical, and magnetic properties of the photospheric layers.

\section{2D Numerical simulations}

We compare two sets of simulations, both with $T_{\text {eff }}=5690 \mathrm{~K}$, and $\log g=4.44$, but with different chemical composition $([\mathrm{M} / \mathrm{H}]=0$ and $[\mathrm{M} / \mathrm{H}]=-2)$. All models are computed on the same 2D Cartesian grid $(400 \times 165$ cells, $\Delta x=28 \mathrm{~km}, 12 \mathrm{~km}<\Delta z<28 \mathrm{~km}$, periodic side boundaries and open/transmitting boundaries at bottom/top). Gray radiative transfer with realistic opacities for the respective chemical composition are used together with an appropriate equation of state that accounts for partial ionization of $\mathrm{H}$ and He. Note that the main effect of the reduced metallicity is a general decrease of the opacity; as a consequence, the gas pressure at the visible surface $(\tau=1)$ is about 3 times higher in the metal-poor model. Figure 1 shows the equipartition magnetic field strength $B_{\text {eq }} \equiv \sqrt{4 \pi\left\langle\rho \vec{u}^{2}\right\rangle}$ (where $\left\langle\rho \vec{u}^{2}\right\rangle / 2$ is the horizontally averaged kinetic energy density) on the geometrical and optical depth scale, as computed from the two non-magnetic simulations.

A homogeneous unipolar vertical magnetic field of four different strengths $\left(B_{\mathrm{z}, 0}=10\right.$ (quiet) 20, 40, and 80 (network) Gauss, respectively, was superimposed on the nonmagnetic models, defining the initial conditions for a series of ideal simulations that are 

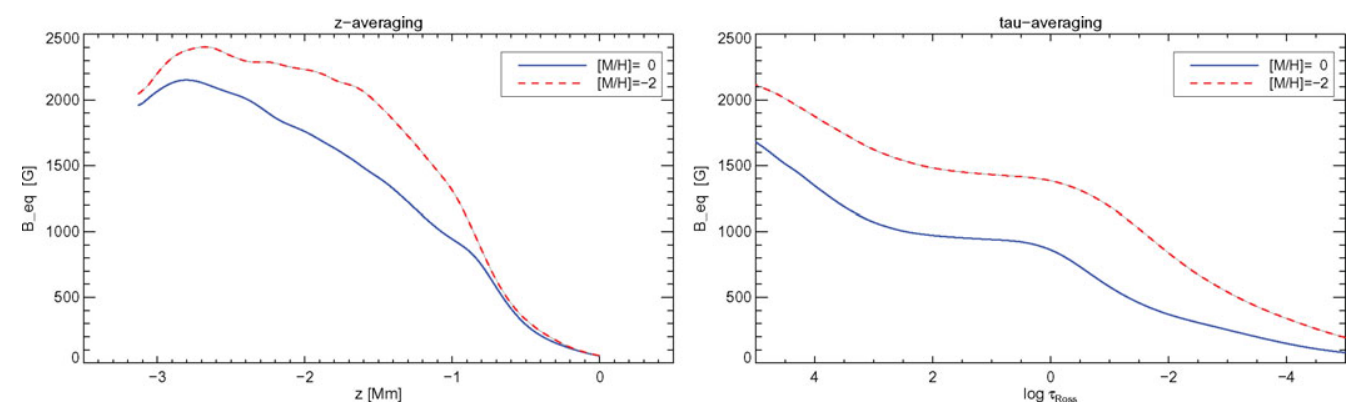

Figure 1. Equipartition field strength, $B_{\text {eq }}$ (see text), on the geometrical height scale (left) and on the optical depth scale (right), computed from the two non-magnetic simulations.
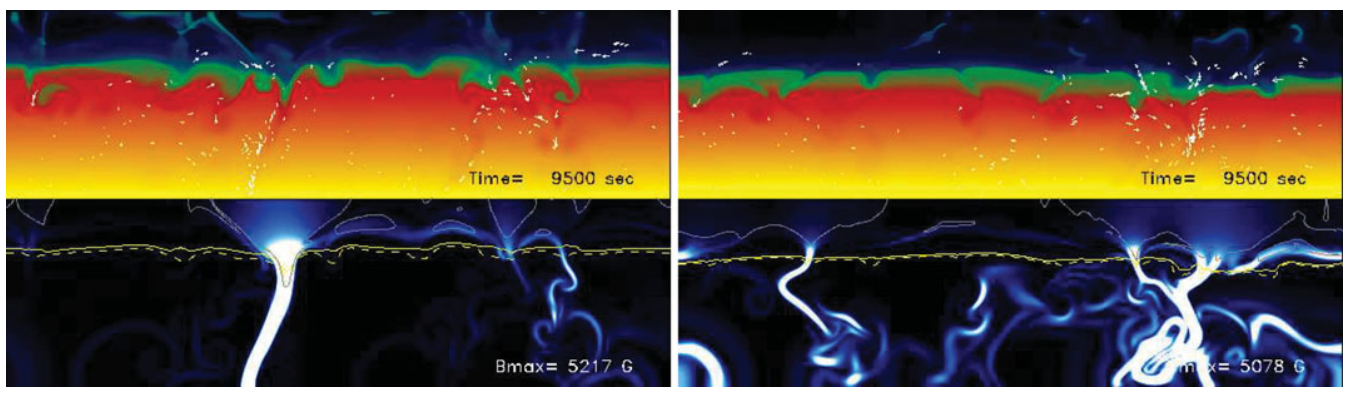

Figure 2. Snapshots from 2D MHD simulations with solar (left) and metal-poor (right) composition, taken $9500 \mathrm{~s}$ after inserting the initial magnetic field $\left(B_{\mathrm{z}, 0}=80 \mathrm{G}\right)$. Upper panels: temperature structure, lower panels: distribution of magnetic field density $|B|$; the dashed (yellow) line delineates the $\tau=1$ contour, gray lines are $\beta=1$ contours. $|B|_{\max } \approx 1.5 \mathrm{kG}$ at $\tau=1$.

used to study near-surface magneto convection at different metallicity as a function of the prescribed mean magnetic flux. At top and bottom, the magnetic field is forced to be vertical $\left(B_{\mathrm{x}}=B_{\mathrm{y}}=0\right)$; at each height, the mean vertical magnetic flux thus retains its initial value $\left\langle B_{\mathrm{z}}\right\rangle=B_{\mathrm{z}, 0}$ throughout these ideal $(\eta=0)$ MHD simulations.

An illustration of the resulting magnetic filed configurations is given in Fig. 2. A first inspection of the simulation data suggests that, for given $B_{\mathrm{z}, 0}$, the rms vertical field $B_{\mathrm{z}, \mathrm{rms}}$ evaluated at fixed optical depth is very similar for the two metallicities, even though $B_{\text {eq }}$ is significantly different (Fig. 1). However, there is a tendency for stronger horizontal fields in the photosphere of the metal-poor simulations.

\section{Conclusions}

These preliminary results need to be confirmed by more extensive $2 \mathrm{D}$ and $3 \mathrm{D}$ simulations with non-gray radiative transfer and alternative MHD boundary conditions. In the long run, further questions can be addressed by computing synthetic (Stokes) spectra based on the MHD model atmospheres, e.g. concerning the influence of small-scale surface magnetic fields on the micro-variability of the stellar radiation and/or on the accuracy of spectroscopic abundance determinations.

\section{References}

Carlsson, M., Stein, R. F., Nordlund, Å., \& Scharmer, G. B. 2004, ApJ 610, L137

Schüssler, M. \& Vögler, A. 2008, A\& $A$ 481, L5

Stein, R. F., \& Nordlund, Å. 2006, ApJ 642, 1246

Steiner, O., Rezaei, R., Schaffenberger, W., Wedemeyer-Böhm, S. 2008, ApJ 680, L85

Vögler, A., Shelyag, S., Schüssler, M., Cattaneo, F., Emonet, T., \& Linde, T. 2005, A\&\&A 429, 335 